\title{
Lusioersily
}

\section{Qualitative evaluation of the SMART2 self-management system for people in chronic pain}

Duggan, G., Keogh, E., Mountain, G., McCullagh, P., Leake, J., \& Eccleston, C. (2015). Qualitative evaluation of the SMART2 self-management system for people in chronic pain. Disability and rehabilitation. Assistive technology, 10(1), 53-60. https://doi.org/10.3109/17483107.2013.845696

Link to publication record in Ulster University Research Portal

\section{Published in:}

Disability and rehabilitation. Assistive technology

\section{Publication Status:}

Published (in print/issue): 01/01/2015

DOI:

10.3109/17483107.2013.845696

\section{Document Version}

Publisher's PDF, also known as Version of record

\section{General rights}

Copyright for the publications made accessible via Ulster University's Research Portal is retained by the author(s) and / or other copyright owners and it is a condition of accessing these publications that users recognise and abide by the legal requirements associated with these rights.

\section{Take down policy}

The Research Portal is Ulster University's institutional repository that provides access to Ulster's research outputs. Every effort has been made to ensure that content in the Research Portal does not infringe any person's rights, or applicable UK laws. If you discover content in the Research Portal that you believe breaches copyright or violates any law, please contact pure-support@ulster.ac.uk. 


\title{
Qualitative evaluation of the SMART2 self-management system for people in chronic pain
}

\author{
Geoffrey B. Duggan ${ }^{1}$, Edmund Keogh ${ }^{2}$, Gail A. Mountain ${ }^{3}$, Paul McCullagh ${ }^{4}$, Jason Leake ${ }^{2}$, and Christopher \\ Eccleston $^{2}$ \\ ${ }^{1}$ Department of Psychology, University of Exeter, Exeter, UK, ${ }^{2}$ Centre for Pain Research, University of Bath, Bath, UK, ${ }^{3}$ School of Health and Related \\ Research, University of Sheffield, Sheffield, UK, and ${ }^{4}$ School of Computing and Mathematics, University of Ulster, Ulster, UK
}

\begin{abstract}
Purpose: Technology could support the self-management of long-term health conditions such as chronic pain. This article describes an evaluation of SMART2, a personalised selfmanagement system incorporating activity planning and review, feedback on behaviour- and acceptance-based therapeutic exercises. Method: The SMART2 system was evaluated over a four-week trial in the homes of people in chronic pain. At conclusion, participants were interviewed to understand the experience of using and living with the SMART2 system as a therapeutic tool. Results: Qualitative analysis of the interviews found that participants liked the system and reported making associated changes to their behaviour. Goal setting and feedback were the most useful elements of the system. A third key and unexpected element was that by simulating some of the functions of a therapist, SMART2 also simulated some of the process of interacting with a therapist. Conclusions: People in chronic pain may experience positive outcomes when using a self-management system designed for behaviour change. Furthermore, some of the supportive aspects of the therapeutic context can be elicited by self-management technologies.

- Implications of Rehabilitation

- Self-management technology has the potential to assist rehabilitation by supporting goal setting and providing feedback.

- By simulating some of the functions of a therapist, technology can simulate some of the process of therapy during rehabilitation.

- People in chronic pain liked using the technology in their own home and thought it could augment services delivered by clinical practitioners.

- Complex programmes of therapeutic exercises delivered by technology had limited success in engaging people in chronic pain.
\end{abstract}

\author{
Keywords \\ Assisted living technologies, E-health, \\ telemedicine
}

\section{History}

Received 25 April 2013

Revised 26 August 2013

Accepted 13 September 2013

Published online 10 October 2013

\section{Introduction}

Chronic persistent pain has major costs to individuals, families and societies. The prevalence of moderate and severe chronic pain associated with major disability is nearly one in five and expected to rise as populations age [1]. However, the range of patient management options is often narrow with limited access to evidence based psychological treatments [2]. Communication technologies could ameliorate this problem through home-based self-management systems that support and guide behaviour change.

Behaviour change interventions are well suited to remote technology-assisted delivery because they are essentially expertbased therapies delivered through guided conversation [3].

Address for correspondence: Geoffrey Duggan, Department of Psychology, University of Exeter, EX4 4DQ, UK. E-mail: gd268@exeter.ac.uk
They rely heavily on features of human exchange, including the use of challenging appraisals, habit reversal, practice, metaphor and behaviour monitoring. All of these features could be replaced, augmented or enhanced using pervasive computer technologies [4]. In addition, technology enables easy record keeping and review, can relieve/complement time-limited expert therapists and could radically increase access to treatments previously inaccessible [5].

Increasingly, interventions are being developed to exploit the potential of communication technology; however, the evidence base on the efficacy of telemedicine is inconclusive [6-8]. More encouragingly, there is evidence that internet-based cognitive behavioural therapy for chronic pain can provide clinically significant improvement in pain intensity [9-11]. Critical to generalising this success to all assisted living technologies, and in improving existing interventions, is to understand how 
these interventions work and which aspects of conventional faceto-face therapy translate most effectively to technology-assisted delivery.

Some progress has been made in understanding behaviour change through Internet interventions [12]; however, there is much work still to do, not least in applying clinical knowledge to the development of technology. We have developed a personalised self-management system for people in chronic pain labelled ' 'SMART2', (www.thesmartconsortium.org). Following inclusive design principles [13], the development process has included extensive consultation with people in chronic pain and clinical professionals and the system incorporates several different components each designed to support self-management [14].

Following the guidance of the Science Panel on Interactive Communication and Health [15], we now report a process evaluation of the SMART2 system. Our dual aim is to inform further iterations of the SMART technology and similar prototypes, and to understand the experience of interacting with selfmanagement technology when in chronic pain. Studying this experience in depth and in situ helps identify which of the components of SMART2 are most likely to be effective and, crucially, to understand how these components work and how they could be improved.

Given the importance of understanding the chronic pain user's experience, we adopt a primarily qualitative methodology. Qualitative analysis is suited to process research as it supports the inquiry into how different elements interact and the interpretation of the meaning of these interactions [16]. To increase the ecological validity, the evaluation was carried out in the homes of people in chronic pain.

\section{Method}

\section{Participants}

Participants were 10 patients ( 5 female) with a mean age of 50.3 years $(S D=12.81$; range $33-75$ years). History of chronic pain was prolonged, with a mean of 14 years $(S D=11.81$; range 5-41 years). The primary complaints of pain were lower back (4 participants), fibromyalgia (3 participants), arthritis (2 participants) and shoulder (1 participant). Nine of the participants reported pain in more than one area. Most participants were not working due to pain, seven participants did not work at all, two worked part-time and one worked full-time.

Inclusion criteria for the study were that participants were over 18 years of age, English literate, had experienced consistent pain for three months or more, were able to walk and wanted to participate in walking-based activity, had adequate dexterity to use mobile phone and computer touchscreens, had no comorbidity or cognitive impairment which would act as a barrier to safe and appropriate participation, and did not suffer with a physical or psychological health problem that realistically could be exacerbated by the use of a pain self-management system (vulnerable populations were excluded).

Recruitment to the study used convenience sampling from two sources. Three patients were recruited from an expert patient panel administered by the University of Bath. For the remainder of participants, a set of 40 patients who had suffered from chronic pain for over six months were identified by General Practitioners and contacted by letter. The first seven patients to reply were included in the study. The study was approved by the UK, NHS National Research Ethics Service (Ref: 08/H1306/46).

\section{The SMART2 system}

The SMART2 system comprises a fixed HomeHub (a Dell Inspiron One 2205 touchscreen computer) and a Mobile Device (an HTC HD2 telephone). Users interact with custom written software that is preloaded on to both devices. The system acquires activity information automatically using accelerometers and GPS sensing and requires patient input to provide additional context.

Here, we provide a brief overview of the different components of the system, more details are provided in [14]. The HomeHub contained five sections: "My Daily Plan", "My Progress", "Information and Advice", "My Maps and Travel", and "My Review'. Screenshots from the first three sections are provided in Figure 1. My Daily Plan showed the goals and activities identified previously by the participant in the goal identification session. Each day participants could select up to four activities with a time at which they wanted to complete them. The My Progress section fed back graphical representations of daily stepcount (derived from accelerometry data), mood and pain levels over the last week. The Information and Guidance section provided eight different audio files of exercises from Acceptance and Commitment Therapy [17] and interactive on screen textboxes for entering responses to the exercises. The acceptance-based exercises were recorded by a clinical psychologist experienced in working in pain management. My Maps and Travel showed a trail of users' location (recorded using GPS) for each activity attempted. My Review provided interactive textboxes where users could record thoughts about their goal-directed activity.

The Mobile Device displayed the activities selected to complete that day and the current daily stepcount. Users selected an activity when they wanted to complete it and recorded their success or otherwise and their pain and mood levels both before and after the activity. A screenshot is provided in Figure 2. The Mobile Device provided an auditory and tactile alert when an activity was due to start and when participants deviated significantly above or below their target stepcount levels. The HomeHub and Mobile Device synchronised automatically and shared information about activity levels, goal setting and goal completion. All interactions with the HomeHub were recorded and timestamped.

A keyboard was used for some responses to the therapeutic exercises and for entering responses into the "My Review" section. All other interactions with the system were by touching the screen on the HomeHub or the Mobile Device.

A day in the life using SMART2 would start with the participant selecting the activities that they would like to complete that day on the HomeHub. For example, to go swimming, visit a friend and vacuum the house. They would then carry the Mobile Device with them in a pocket or belt pouch. At the selected time the Mobile Device would alert them that an activity was due, they would rate their pain and mood on the Mobile Device and record that they had started the activity. Upon completion of the activity, they would record the outcome of the activity and their pain and mood levels. The Mobile Device would then provide some outcome appropriate feedback. If at any point in the day they were over- or under-exercising, the Mobile Device would alert them and enable them to modify their activity level. At the end of the day (or at any other point), the participant could use the HomeHub to review their levels of exercise, pain and mood over the last week and the number of activities completed. They could then record any comments in the My Review section and complete a guided therapeutic exercise.

\section{Questionnaire measures}

To complement the qualitative analysis self-report scales were also completed by participants, these were the Brief Pain Inventory [18], Chronic Pain Acceptance Questionnaire [19] and the System Usability Scale [20]. The extent to which users found using the system in their home acceptable and could be 

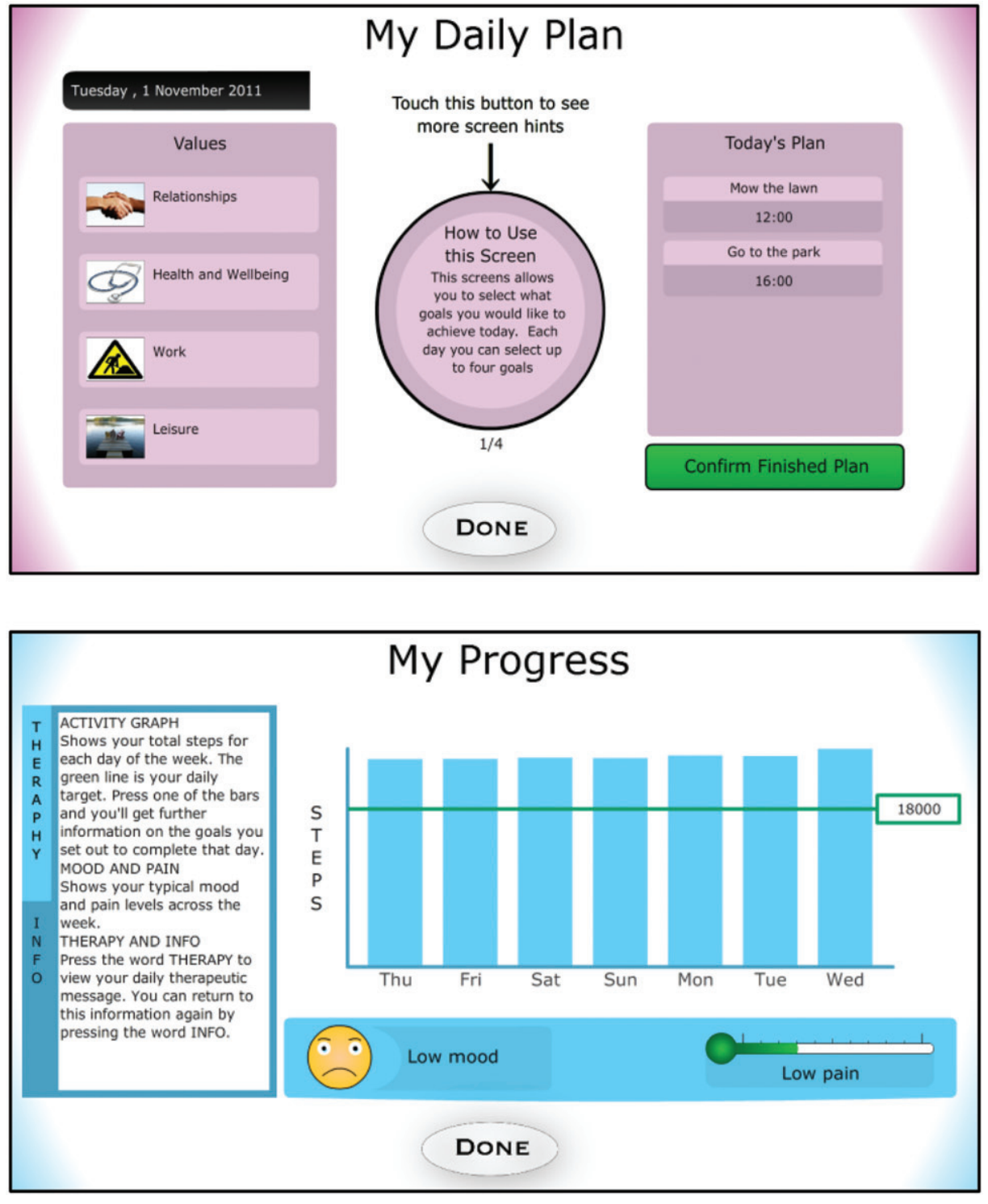

\section{Information And Advice}

\section{Welcome}

This is the therapy exercises section of the system. These exercises are not physical exercises, instead, they are psychological exercises designed to help you relate differently to your thoughts and experience. Each of the boxes below contains a different exercise for you to have a 90 at. At different points during your use of the system a new exercise will become available - you will be informed when a new exercise is available on the 'My Progress' page.

The exercises will be presented through an audio recording. You may also be asked to type in a response using the keyboard. The exercises may seem a little odd at they suggest.

Once you have completed an exercise you can go back and repeat it or reread your response any time you want. You may want to listen to or practice one of the exercises again.

\begin{tabular}{|c|}
1 \\
\hline $\begin{array}{c}\text { Introduction to } \\
\text { the therapeutic } \\
\text { exercises }\end{array}$ \\
\hline
\end{tabular}

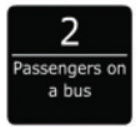

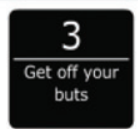

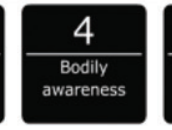

\section{Audio}

Audio is started by pressing the speaker icon which looks like this:

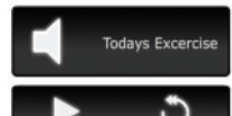

You can stop, pause and replay the audio by pressing the buttons.

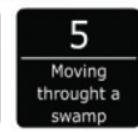

$\frac{6}{\substack{\text { Noticing } \\ \text { yourself }}}$
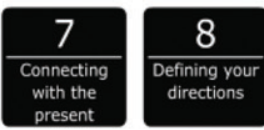

\section{DONE}

Figure 1. Screenshots from the My Daily Plan, My Progress and Information and Advice sections from the HomeHub. 


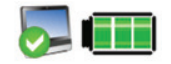

\section{Today's Goals}

\begin{tabular}{|l|l|}
\hline Total Steps & Target \\
\hline 10012 & 20000 \\
\hline
\end{tabular}

Go to the park

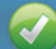

Walk the dog

Figure 2. Screenshot from the Mobile Device showing the homepage with one goal completed and one goal still to be completed.

feasibly incorporated into their everyday life was assessed using a scale adapted from [21]. Knowledge of pacing was tested by asking participants to respond "Agree", "Disagree" or "Don't know" to 15 statements about pacing.

\section{Procedure}

A researcher trained in goal setting visited the participants in their homes on three occasions. At the first meeting, the researcher and participant collaboratively identified participant goals from four categories: health and well-being, work, leisure and relationships. These categories are used in goal setting within Acceptance and Commitment Therapy [17]. Participants were encouraged to generate as many goals as possible with at least one goal from each category. Concrete activities related to these goals were then listed (i.e. the goal "Improve Fitness" could include activities like "Swimming", "Stretching Exercises"). Participants also completed the Brief Pain Inventory, the Chronic Pain Acceptance Questionnaire and the Pacing Knowledge Questionnaire. They were given the Mobile Device, instructed in the use of the device and asked to carry it with them at all times to establish a baseline daily stepcount. A target number of steps to be completed every day was selected by the participant as a proportion of their baseline daily stepcount.

One week later, the researcher visited the home again with technical support and installed the complete SMART2 system. This involved installation of the HomeHub PC, a standard wireless router and configuring the Mobile Device and HomeHub for communication. Participants then received a full demonstration with instructions and practised using the system. A user manual, email address and telephone contact number were provided for technical support. The third visit was four weeks after the second visit. The system was collected and participants completed all of the self-report scales before being interviewed.

\section{Post-intervention interviews}

Semi-structured interviews lasting approximately 40 minutes were conducted in a 1-to-1 setting. Interviews were guided by a structured protocol of open-ended questions with follow-up probes. The protocol explored the following topics: usability, pacing, personalisation of system, pain level and system use, acceptance-based therapeutic exercises, goal setting and longterm behaviour change. All interviews were audio recorded.

Experimenter bias was limited through the use of a semistructured interview protocol and by emphasising to participants
Table 1. Response Means (SD) for participants who completed trial $(N=8)$.

Pre-intervention Post-intervention

BPI

Pain Intensity

Pain Interference

CPAQ

Activity Engagement

Pain Willingness

Total

Pacing Knowledge

SUS (0-100)

Acceptability and Feasibility (1-10)

$5.05(1.44)$

$5.38(1.13)$

$5.37(2.58)$

$5.89(1.51)$

$37.40(9.02)$

$37.88(12.79)$

$22.90(8.95)$

$24.39(8.30)$

$60.30(15.53)$

$62.25(18.87)$

$0.59(0.23)$

$0.63(0.24)$

$66.25(12.25)$

$5.38(1.74)$

$\mathrm{BPI}=$ Brief $\quad$ Pain Inventory, $\mathrm{CPAQ}=$ Chronic $\quad$ Pain Acceptance Questionnaire, SUS $=$ System Usability Scale.

the exploratory nature of the study and that we were keen to hear both positive and negative comments. Experimenter bias within the thematic analysis was lessened by including three different analysts, one of which was independent to the study.

\section{Analytic approach}

A thematic analysis of the data obtained in the semi-structured interviews was conducted following the guidelines provided by [16]. The primary analyst (GD) transcribed interviews and then read them in their entirety before generating initial codes and using these to code each of the interviews. These codes were grouped into themes and subthemes and all relevant coded data extracts were collated within these themes. Themes were then reviewed and their validity was considered in relation to the meaning in the data set as a whole. A second analyst (JL) read through the complete transcripts for two interviews (25\% of the data) and recoded the data with a high level of interrater reliability $(K=0.74, S E=0.06)$. Finally, the entire thematic analysis was reviewed by a clinical psychologist experienced in working in pain management and unconnected with the study. Themes were corrected and elaborated following discussion with GD.

\section{Results}

Two participants stopped the trial during the baseline condition in the first week. One was due to deterioration in her pain condition (arthritis) that was not attributed to the trial; the other found the mobile device too heavy and was too busy during the trial period.

The self-report scales provide a profile of the participants preand post-intervention. These scores and the measures of usability were not analysed statistically. The primary analysis was the thematic analysis of the interviews.

\section{Descriptive measures}

Mean scores for the quantitative measures are given in Table 1. Scores pre- and post-intervention were similar for all measures. There was a change in CPAQ score for one participant (Participant 08: Pre-intervention $=59$, Post-intervention $=79$ ). Usability and acceptability scores were around the midpoint of both scales. During the 28-day trial period, the mean number of days that participants used the system was $20(S D=6.76)$ and the mean number of HomeHub touchscreen presses was 1448.25 $(S D=983.73)$. The mean number of target activities generated by the eight completing participants during the goal identification session was $31.50(S D=4.78)$. Technical difficulties meant that reliable measures of stepcounts and goal completion were not recorded. Across all participants, there were 15 calls and 2 emails requesting technical support. 


\section{Thematic analysis}

Five different themes were identified from the thematic analysis: Technical Difficulties and Beneficial Effects, Goal Setting, Feedback, Therapeutic Content and Process of Behaviour Change. Each theme is described below and the subthemes are specified with an example extract in Table 2. Extracts were selected to illustrate the subthemes and represent all of the participants. Where there were conflicting opinions an extract for each opinion is provided. For key subthemes, multiple extracts are provided to provide a richer explanation of the subtheme.

\section{Technical difficulties and beneficial effects}

The participants stated that the SMART2 interface was attractive and many enjoyed using the touchscreen on the HomeHub which for some acted as an incentive to use the system (Q1). They thought the interface was well designed and, when functioning,

Table 2. Themes and subthemes from the qualitative analysis of post-intervention interviews. Example extracts are provided for each subtheme.

$\begin{array}{ll}\text { Themes } & \text { Examples }\end{array}$

Technical Difficulties and Beneficial Effects

Attractive interface

Ease of use

Frustration with technical difficulties

Beneficial effects of system

\section{Goal Setting}

Increased planning

Provided structure

Increased achievements

Broadened scope of activity

Pacing through goal setting

Feedback

Reinforcement for goal completion

Frustration at lack of reinforcement

Value of alerts

Value of reviewing activity

Pacing through feedback

Loss of trust

Therapeutic Content

Simulated Therapist

Unsuccessful therapeutic exercises
Q1 “The look is a nice clean elegant look. It's a clean elegant system”. Participant 01.

Q2 "It was really easy to use, anyone could have used it". Participant 10.

Q3 "I found it frustrating there were lots of problems". Participant 03.

Q4 “Aside from the usability I think it's a great system. I would definitely use it". Participant 01 .

Q5 “It has a beneficial effect on your general life and the way you're living and the way you're thinking about yourself and other people too'. Participant 05.

Q6 "I think going forward it would be great to have it permanently". Participant 02.

Q7 "This is getting me to do stuff and have a rewarding life... I really loved the trial. I'd buy the app". Participant 08 .

Q8 “'It did make you plan for the future so you were thinking ahead. . it added some structure to your day". Participant 05.

Q9 "I feel a bit at sea at the moment because I've got no goals, because this [the trial] has ended". Participant 04.

Q10 "I did find that very useful actually - setting the goals every morning. I found that I was achieving more than I think I would without having those goals set'”. Participant 02.

Q11 "I did more different things which made my quality of life better and made me think about the different things. Yeah, I was pretty pleased". Participant 05.

Q12 "It [goal setting] does make you pace yourself and think more about what you're doing each day". Participant 07.

Q13 “And that's the thing, you gain satisfaction just by entering it. Which I didn't see that at the beginning, I just thought that you need a reward. But the reward is actually being able to say 'I've done it"'. Participant 01.

Q14 'If I looked back for a day when it hadn't connected it would come up with 4 red marks saying I hadn't done it and I'd find that really, really demoralising''. Participant 08.

Q15 'It did make you stop and think, so that's probably where I do need reminding at times'. Participant 04

Q16 'Sometimes you don't realise you've done that little bit extra until you actually see it in front of you and you think 'aha that's why' [there is pain]'. Participant 07.

Q17 'It did work and I didn't find myself having these sort of days when I'm like, 'Oh my God I've got to do this' and hammering through it all day and collapsing in a heap for three days afterwards'. Participant 05.

Q18 "I had a quick look at that and the feedback but generally I think I lost trust in it because I knew. . . it hadn't recorded everything', Participant 01.

Q19 'I've got carers and they motivate me for that half an hour in the morning and half an hour in the evening. But the rest of the time, there's the odd friend will do something erratically but I need something every single day and something with me like that thing [points at SMART2 mobile] to motivate me because I haven't got a parrot on my shoulder saying 'Come on you can do this, come on you can do this"'. Participant 08 .

Q20 "It's almost having someone checking up on you if you like. Just makes you more likely to think, 'yeah, I will go and do it' rather than 'I'll just sit in front of the telly','. Participant 02.

Q21 "Sometimes you need that little bit of help just to pick you up, so that's where the idea of this, that if there was something there that sometimes I could say, 'ooh, I'm getting down into that circle', you don't want to get to the bottom of that big pit, if you can just pull yourself up'. Participant 04 .

Q22 “As soon as you start putting that pain in, you start noticing it and if it doesn't acknowledge it [due to technical failure], then suddenly it's like 'I'm suffering and no one's acknowledging it'". Participant 01

Q23 'The whole system gave me a nice incentive to do something and when I' $m$ at home all the time it's so easy to fall into that crevice where you don't want to do anything and that's it you're a slob. Where you can get something like that and it pulls you out slightly and then you might do another thing and it pulls you out a bit more. So it is a good thing to do, the system. It can pull you out of that little hole you're in, I definitely believe that and it could get you start moving again'. Participant 10.

Q24 "Like a nagging wife but in a good way". Participant 05.

Q25 "I didn't feel the need for the content that I listened to". Participant 03. 
Table 2. Continued

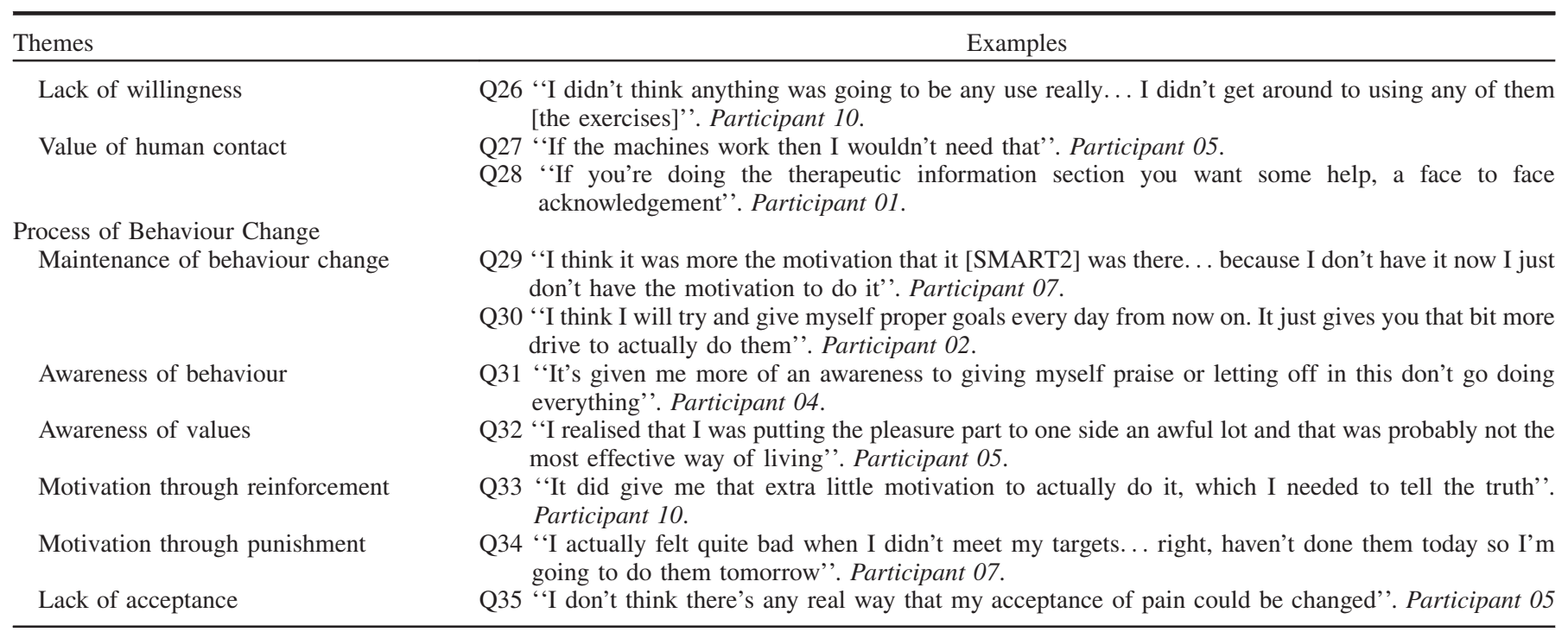

was easy to use (Q2). However, there were technical difficulties with the system. The most common problems were a lack of consistent connection between the Mobile Device and the HomeHub and system failures to record responses or stepcounts. All participants reported that these problems significantly disrupted their user experience and led to considerable frustration with the system (Q3). The combination of positive feedback on the interface design and the frequency of technical problems accounted for the mid-range scores on the System Usability Scale and Acceptability and Feasibility Scale in Table 1.

All participants reported that the technical difficulties affected their perception of the system but that they also managed to trial elements of the system on some days. One of the most striking results from the trial was that despite the technical difficulties and consequent frustrations, seven out of eight participants stated that the system had a beneficial effect on their life and some participants made important changes to their behaviour. Further, participants were enthusiastic about the future potential for the system (Q4-Q7). The remaining four themes identify those elements that led to the positive changes in participants.

\section{Goal setting}

Goal setting occurred both during the initial goal identification session and when constructing a plan at the start of the day. Participants stated that both activities increased planning and provided structure (Q8, Q9). Constructing a daily plan led to participants achieving more and focusing on the activities that were most important to them (Q10).

In part, these improvements were due to the therapeutic structure incorporated into SMART2. During the goal identification session participants were asked to generate goals for different categories from their life and these goals were deconstructed into smaller activities. Having goals that corresponded to different areas of their life was very helpful for some participants and they set and completed goals they would not ordinarily have attempted (Q11). Constraining participants to four activities per day helped them to break down superordinate goals into more manageable tasks and supported pacing (Q12).

\section{Feedback}

The process of entering the outcome of one of the daily activities and then seeing it stored on the system reinforced goal-directed behaviour and acted as a reward for the participants. This feedback augmented the benefits from setting goals (Q13). The importance of having the system record behaviour was demonstrated by the disappointment and frustration from participants when technical failures meant goal successes were not recorded (Q14).

To support pacing, participants received alerts when their stepcounts were particularly low or high and were able to review daily stepcounts and levels of pain and mood over time. This feedback was heavily impaired by technical difficulties; however, to the extent that it worked, participants thought it useful (Q15). The interruption of the alerts drew participants' attention to their activity levels and participants enjoyed reviewing their behaviour and used it to identify patterns that led to greater pain (Q16). Most of the participants did not find their pacing improved but thought more reliable feedback would help their pacing (Q17).

The effectiveness of feedback was dependent on the extent to which participants trusted the system. When participants stopped trusting the system even accurate feedback was of limited use (Q18).

\section{Therapeutic content}

Therapeutic content refers to the use of the specific acceptancebased therapeutic exercises as well as the general sense of therapeutic interaction that many participants reported. This sense of a "simulated therapist" was an important subtheme not anticipated prior to the evaluation. The importance of trust and the reinforcement received from recording completed goals on the system are also indicators of this subtheme. In short, it appeared that the process of interacting with an external agent had a therapeutic benefit in and of itself. Participants spontaneously described the system as like "'a personal trainer', “'a nagging wife" or "a parrot on the shoulder'. They reported that interacting with the system helped to break negative cycles of thought and crucially, unlike human support, the system was always available. SMART2 was not designed to provide empathy but interacting with it raised awareness and motivation and simulated some aspects of a conventional human therapist interaction (Q19-Q24).

The recorded therapeutic exercises based on Acceptance and Commitment Therapy were not successful for most participants and only one participant completed all the exercises and claimed much benefit (Q25). Participants reported that the content was not 
very relevant to them or engaging. There was also a lack of willingness to try the exercises despite strong encouragement to do so at the start of the trial (Q26).

Participants were divided about the value of including more contact with a human within the system. Some felt that it would be an incentive to use the system generally and could help explain the therapeutic exercises whereas others felt it was not necessary (Q27, Q28).

\section{Process of behaviour change}

The final theme related to the maintenance of behaviour change and the changes in the participants that led to behaviour change during the trial. Participants were divided about the likelihood of maintaining any behaviour changes made during the trial. Some felt these changes would have a lasting impact on their behaviour irrespective of having the system, whereas others felt the changes were dependent on having the system (Q29, Q30).

Participants reported an increase in awareness and motivation. Goal setting, feedback and interacting with the system all increased awareness of day-to-day behaviour both at the time of the behaviour and in review (Q31). These three elements also increased participants' awareness of their values and helped identify the behaviours most important to participants (Q32).

Participants also reported feeling more motivated to carry out value consistent behaviour. This motivation was increased by goal setting, feedback and interacting with the system. Primarily, these elements provided reinforcement or incentives (Q33); however, feedback on failing to complete a goal or on achieving the desired level of activity acted as a punishment and increased motivation (Q34).

Seven participants reported no change in their acceptance of pain (Q35). Participant 08 reported an increase in acceptance and her CPAQ score increased. She was also the only participant to report any benefit from the therapeutic exercises.

\section{Discussion}

This evaluation investigated the experience of someone in chronic pain interacting with SMART2 to understand which elements of the system could be most effective and to develop a greater understanding of how technology could be designed to support self-management. In line with this aim, the results provided greater support for some aspects of the system than others. The primary analysis was qualitative and this found that the participants liked many aspects of the SMART2 system and felt that it led to positive changes in their behaviour. The key elements that were successful and the relationships between these elements are summarised in Figure 3.

Figure 3 represents that supporting goal setting, providing feedback and simulating a therapist were all functions of SMART2 that participants reported were helpful, and that each of these elements reinforced the effectiveness of the others. Goal setting helped identify activities that were important to participants and provided structure to participants' lives. Feedback from the system reinforced goal-directed behaviour and also enabled participants to pace themselves through goal setting and by providing information about activity levels. The third key element was that the system was an external agent that interacted with participants. By simulating some of the functions of a therapist, SMART2 also simulated some of the process of interacting with a human therapist and this process was helpful to participants.

Participants reported that each of these three elements interacted with one another. Figure 3 extends these observations to describe the relationships between each of the three elements when using SMART2. Goal setting provided opportunities for feedback on goal completion and feedback reinforced future

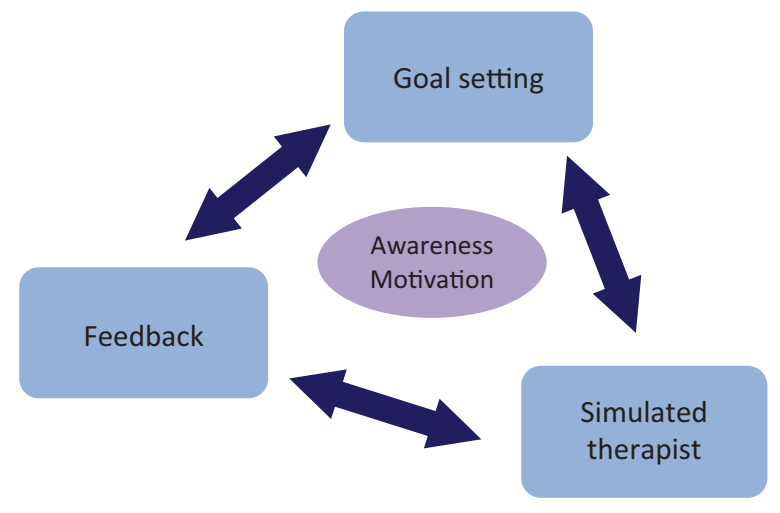

Figure 3. The three key elements of SMART2 that supported behaviour change and the proposed relations between these elements. Awareness and motivation are included within these three elements as the mechanisms described by participants as leading to behaviour change.

goal setting. Setting goals and receiving feedback underpinned the experience of simulating a therapist interaction. Moreover, an increased sense of interacting with an external agent, increased participants' tendency to set goals and increased the value of feedback. Participants reported that behaviour change occurred by increasing their awareness of themselves or their behaviour and by increasing their motivation to carry out value consistent behaviour.

The observation that goal setting and feedback can lead to behaviour change is not new [22]. Our results are interesting because they show that technology could potentially be used to support goal setting and feedback and that this can help people in chronic pain using the technology unsupervised in their own homes. This is noteworthy given the limited success of many selfmanagement interventions [23-25]. Further, the thematic analysis stipulates the ways that goal setting and feedback were helpful which could act as a template for other self-management interventions that exploit the ubiquity and interactivity of communication technologies.

Figure 3 indicates that the experience of interacting with an external agent or "simulated therapist"' was helpful. In a systematic review of technologies leading to behaviour change, Rosser et al. [26] concluded that human involvement within an intervention was an important predictor of dropout rates. We extend that conclusion to suggest that replicating aspects of human interaction could improve the engagement with selfmanagement interventions. Achieving this is difficult with the paper-based systems that are typically used within self-management; however, technology offers new possibilities [27], for example, by linking users together through peer communities. In our study, participants spontaneously likened SMART2 to things that have agency and reported that this was helpful. They also reported that this encouraged them to set goals and respond to feedback.

Understanding how to helpfully simulate aspects of human interaction goes beyond the scope of this article, but we note that SMART2 did neither use any human-like presentational features such as names or graphics, nor did it attempt to provide empathy. Rather, it was personalised and much of the interaction centred on meaningful goals and activities (see [28] for further discussion of anthropomorphism).

One of the least successful sections of the system was the acceptance-based therapeutic exercises. Audio recordings with on-screen textboxes to enter feedback did not engage the participants. Thus, most participants were reluctant to try the exercises and gained little benefit from them. It appeared that this section failed to simulate many of the important features of a 
human therapist interaction. The lack of engagement may be improved through a more sophisticated implementation of therapeutic exercises (e.g. greater use of multimedia and the inclusion of human prompts). These methods have been effective within other technology-based treatment programmes [10].

\section{Limitations and future directions}

An important constraint on the evaluation was the technical difficulties experienced by all participants. Technical difficulties were identified in two areas: (1) changes in communication parameters such as IP address and communication sockets over time and (2) unanticipated use of the touchscreen to prematurely terminate a recording. These issues have been addressed by software updates but during this evaluation limited the extent to which we could accurately measure the impact of feedback on behaviour and also degraded participants' trust in the system as a whole. Nonetheless, participants were able to successfully trial many aspects of the system in a home environment and the changes reported by some participants were even more impressive given these difficulties.

Participants were an opportunity sample who volunteered for the study, and therefore may not be representative of the community chronic pain population. Nonetheless, any selection bias was counteracted by the selection of participants with different pain conditions and by recruiting from different sources. Limitations on the generalisability of the findings are usual at this stage of evaluation [15], which focuses on the feasibility of the system, and the detail of frequent use.

Three main findings can be incorporated into future investigations of a self-management system for chronic pain. First, the technical challenges of the deployment of multiple interacting device systems deployed in home environments are not to be underestimated and next generation systems will need to be more robust. Second, investigation of the role of assigned agency is worth serious investigation as a potentially potent mediator of change. Third, the next generation of the SMART2 system should be put to the highest standard of test of a randomized controlled trial with a focus on the efficacy of any system in improving pain, disability and distress.

\section{Conclusion}

By supporting goal setting, providing feedback and acting as an external agent that participants can interact with, technology has great potential for supporting the self-management of chronic pain. The SMART2 system has successfully incorporated these elements within its design. Future research needs to address technical difficulties and ultimately evaluate patient outcomes using a randomized controlled trial.

\section{Acknowledgements}

We thank all the participants who took part in the trial and the members of the SMART consortium (www.thesmartconsortium.org) for their input into the development of SMART2.

\section{Declaration of interest}

The authors report no declarations of interest.

This research was supported by a grant from the Engineering and Physical Sciences Research Council, UK (EP/F001916).

\section{References}

1. Breivik H, Collet B, Ventafridda V, et al. Survey of chronic pain in Europe: prevalence, impact on daily life, and treatment. Eur J Pain 2006;10:287-333.
2. Eccleston C, Williams ACDC, Morley S. Psychological therapies for the management of chronic pain (excluding headache) in adults. Cochrane Database Syst Rev 2009;(2):CD007407.

3. Khan N, Bower P, Rogers A. Guided self-help in primary care mental health: meta-synthesis of qualitative studies of patient experience. Br J Psychiatry 2007;191:206-11.

4. Andersson G. Using the internet to provide cognitive behaviour therapy. Behav Res Ther 2009;47:175-80.

5. Murray E, Burns J, See TS, et al. Interactive health communication applications for people with chronic disease. Cochrane Database Syst Rev 2005;(4):CD004274.

6. Car J, Huckvale K, Hermens H. Telehealth for long term conditions. BMJ 2012;334:e4201.

7. Currell R, Urquhart C, Wainwright $P$, Lewis R. Telemedicine versus face to face patient care: effects on professional practice and health care outcomes. Cochrane Database Syst Rev 2010;(2):CD002098.

8. Wantland DJ, Portillo CJ, Holzemer WL, et al. The effectiveness of web-based vs. non-web-based interventions: a meta-analysis of behavioral change outcomes. J Med Internet Res 2004;6:e40.

9. Bender JL, Radhakrishnan A, Diorio C, et al. Can pain be managed through the internet? A systematic review of randomized controlled trials. Pain 2011;152:1740-50.

10. Palermo TM, Wilson AC, Peters M, et al. Randomized controlled trial of an internet-delivered family cognitive-behavioral therapy intervention for children and adolescents with chronic pain. Pain 2009;146:205-13.

11. Velleman S, Stallard P, Richardson T. A review and meta-analysis of computerized cognitive behaviour therapy for the treatment of pain in children and adolescents. Child Care Hlth Dev 2010;36:465-72.

12. Ritterband LM, Thorndike FP, Cox DJ, et al. A behaviour change model for internet interventions. Ann Behav Med 2009;38:18-27.

13. Newell AF, Gregor P. User sensitive inclusive design - in search of a new paradigm. Proceedings of A.C.M. Conference on Universal Usability. Washington, DC; 2000:39-44.

14. Rosser BA, McCullagh P, Davies R, et al. Technology-mediated therapy for chronic pain management: the challenges of adapting behaviour change interventions for delivery with pervasive communication technology. Telemed J E Health 2011;17:211-16.

15. Eng TR, Gustafson $\mathrm{DH}$, Henderson $\mathrm{J}$, et al. Introduction to evaluation of interactive health communication applications. Science Panel on Interactive Communication and Health. Am J Prev Med 1999;16:10-15.

16. Braun V, Clarke V. Using thematic analysis in psychology. Qual Res Psychol 2006;3:77-101.

17. Hayes SC, Luoma JB, Bond FW, et al. Acceptance and commitment therapy: model, processes and outcomes. Behav Res Ther 2006;44: $1-25$.

18. Cleeland CS, Ryan KM. Pain assessment: global use of the Brief Pain Inventory. Ann Acad Med Singapore 1994;23:129-38.

19. McCracken LM, Volwes KE, Eccleston C. Acceptance of chronic pain: component analysis and a revised assessment method. Pain 2004;107:159-66.

20. Brooke J. SUS: a "quick and dirty usability scale". In: Jordan PW, Thomas B, Weerdmeester BA, McClelland AL, eds. Usability evaluation in industry. London: Taylor and Francis; 1996:189-94.

21. Borkovec TD, Nau SD. Credibility of analogue therapy rationales. J Behav Ther Exp Psychiatry 1972;3:257-60.

22. Locke EA, Latham AP. Building a practically useful theory of goal setting and task motivation. Am Psychol 2002;57:705-17.

23. Warsi A, LaValley MP, Wang PS, et al. Arthritis self-management programs: a meta-analysis of the effect on pain and disability. Arthritis Rheum 2003;48:2207-13.

24. Newman S, Steed L, Mulligan K. Self-management interventions for chronic illness. Lancet 2004;364:1523-37.

25. Griffiths C, Foster G, Ramsay J, et al. How effective are patient (lay led) education programmes for chronic disease? BMJ 2007;334: 1254-6.

26. Rosser BA, Vowles KE, Keogh E, et al. Technologically assisted behaviour change: a systematic review of studies of novel technologies for the management of chronic illness. J Telemed Telecare 2009;15:327-38.

27. Reeves B, Nass C. The media equation: how people treat computers, television, and new media like real people and places. Cambridge: Cambridge University Press; 1996.

28. Epley N, Waytz A, Cacioppo JT. On seeing human: a three-factor theory of anthropomorphism. Psychol Rev 2007;114:864-86. 